\title{
Les risques de pertes indirectes induites par les systèmes informatiques *
}

par André George (Pactel France) **

SOMMAIRE

page

1. INTRODUCTION 58

2. OBJECTIFS DE LA RECHERCHE

3. RESUME DES CONCLUSIONS

3.1 Sécurité générale des systèmes informatiques

3.2 Pertes financières directes

3.3 Pertes indirectes

3.4 Problèmes divers rencontrés pendant l'étude

4. METHODES DE TRAVAIL

4.1 Positionnement du problème

4.2 Enquêtes

4.3 Etude de cas

4.4 Validation qualitative

4.5 Synthèse finale

5. PERTES DIRECTES

5.1 Sécurité générale des systèmes informatiques

5.2 Evaluation des pertes financières directes

5.2.1 Définition des risques

5.2.2 Risques

* Etude réalisée pour le compte de l'« Association de Genève ».

** Avec la collaboration de E. Benejam (Pactel France), H. D. Scholz (Pactel Allemagne), R. R. Strasser (Pactel Suisse) et P. A. Thomas (Pactel Grande-Bretagne). 


\subsubsection{Applications}

\subsubsection{Incidents}

5.2.5 Niveau de risque et type de système informatique utilisé

\subsubsection{Synthèse}

\section{PERTES INDIRECTES}

6.1 Définitions adoptées

6.2 Méthode de travail

6.3 Rappel sur la sécurité générale des systèmes informatiques

6.4 Estimation globale des taux de couverture des pertes indirectes

6.5 Analyse par applications

6.5.1 Amplitudes des pertes indirectes

6.5.2 Synthèse des niveaux de pertes indirectes possibles par type d'application

6.5.3 Fréquence des pertes indirectes

6.6 Analyse par type d'incidents

\subsubsection{Résultats généraux}

6.7 Analyse des niveaux de pertes indirectes par secteurs économiques étudiés

6.8 Analyse des niveaux de pertes indirectes par type de systèmes installés

6.9 Analyse par types de dommages 


\section{Introduction}

L'étude menée en 1976 pour le compte de l'Association de Genève ${ }^{1}$ avait pour objectifs essentiels, dans le cadre européen et à l'horizon 1988 :

- d'évaluer le développement de l'utilisation de l'informatique et d'identifier les principales applications :

- de gestion,

- de contrôle et régulation des processus industriels,

- de type public et social ;

- d'évaluer les risques économiques encourus par les utilisateurs ; utilisateurs étant ici pris au sens large du terme, c'est-à-dire aussi bien les personnes physiques dans l'entreprise ou organisme : employés, informaticiens, que les personnes physiques hors de l'entreprise (clients) et que, bien entendu, les personnes morales : l'entreprise utilisatrice elle-même, les firmes clientes, etc ;

- de proposer les palliatifs envisageables et d'en déduire les moyens (produits/méthodes) que l'industrie de l'assurance devrait mettre en œuvre pour pallier les risques et problèmes identifiés.

Voici un rappel des conclusions de cette étude :

- accroissement considérable de l'utilisation de l'informatique en Europe de l'Ouest dans la période considérée :

- 1973 : 55000 équipements installés pour une valeur de 40 milliards de francs suisses,

- 1988 : 450000 systèmes installés pour une valeur de 200 milliards de francs suisses ;

- progrès technologiques importants ;

- évaluation des dommages économiques annuels causés par des incidents divers dans les systèmes informatiques à environ 12 milliards de francs suisses, pour des incidents dont l'amplitude ira d'une centaine à 28 millions de francs suisses.

Or, Monsieur Orio Giarini insistait, dans sa préface à l'étude, sur deux notions essentielles :

«... Sans aucun doute l'informatique technologique représente un grand potentiel de progrès... Le monde contemporain nécessite plus que jamais le sens de la navigation, qu'on appelle couramment capacité de management... Nous avons donné une nouvelle dimension à tout, y compris à l'incertitude... Il faut qu'un développement aussi important que celui de l'informatique puisse éviter les écueils de l'incompréhension. »

« La douzaine de milliards de francs suisses de dommages annuels, dus à l'utilisation de l'ordinateur en Europe en 1988, représente une estimation de la valeur déduite (...) qui représente un pourcentage supportable de la valeur ajoutée nette produite par le secteur de l'informatique. »

Il est donc apparu opportun d'étudier de façon plus détaillée et concrète le problème posé par les risques inhérents aux pertes indirectes: perte d'exploitation et

1 « Nature et importante des pertes économiques dans l'utilisation de l'informatique en Europe en 1988 », publiée dans «Les cahiers de Genève » no 3, octobre 1976. 
responsabilité civile/produits qui représentent suivant les secteurs économiques et les applications considérées dans la première étude entre 50 et $95 \%$ des pertes potentielles totales.

\section{Objectifs de la recherche}

La recherche entreprise couvre :

- l'Europe de l'Ouest;

- les secteurs de l'industrie, du commerce et des services où les pertes indirectes sont d'un niveau supérieur à celui évalué dans les secteurs publics et para-publics.

Ces trois secteurs ont été segmentés de la façon suivante :

- industrie - process,

- assemblage ;

- commerce ;

- banques - assurances ;

- services divers.

Les objectifs particuliers de cette recherche doivent permettre de déterminer :

- la nature et l'importance des risques de pertes indirectes à couvrir du fait de l'utilisation de systèmes informatiques, en analysant, pour chacun des secteurs économiques considérés, les risques par:

- type de systèmes informatiques utilisés,

- type d'applications réalisées ;

- la possibilité de préparer, pour les professionnels de l'assurance, une méthode d'évaluation rationnelle des risques en fonction des différents paramètres étudiés ;

- l'esquisse d'une politique à mener par les assureurs pour prévenir et réduire les risques, donc la définition des méthodes, thèmes et moyens à mettre en œuvre.

\section{Résumé des conclusions}

La recherche entreprise pour l'Association de Genève, dans les secteurs des finances (banques, assurances), de l'industrie, du commerce et des services en Europe de l'Ouest, a pour objectifs principaux:

- de déterminer la nature et l'importance des risques de pertes indirectes induites par l'utilisation de l'informatique dans l'entreprise ;

- d'esquisser la politique à mener par l'industrie de l'assurance dans ce domaine.

Pour aboutir aux conclusions de l'étude, plusieurs groupes de travail ont été réunis, environ 100 enquêtes ont été effectuées et trois études de cas ont été réalisées. Il nous faut ici exprimer nos plus vifs remerciements à tous ceux qui ont accepté de participer à cette étude en y apportant leur talent et leur temps.

Les principaux points qui se sont dégagés de cette étude sont schématisés ci-dessous.

\subsection{Sécurité générale des systèmes informatiques}

Il paraît nécessaire d'améliorer la sécurité des systèmes informatiques, en particulier dans les secteurs de l'industrie et du commerce, tant sur le plan des systèmes d'informations que sur celui des équipements et des logiciels (programmes). 
Les assureurs doivent, à notre avis, imposer un certain niveau de prévention/ détection des sinistres, notamment sur le plan des procédures de sauvegarde et de dépannage.

Pour y parvenir, nous suggérons :

- une assistance plus effective à leurs assurés et des contrôles de vérification périodiques de la sécurité informatique ;

- une incitation des assurés au niveau de la tarification des primes, à mettre en œuvre et maintenir une politique cohérente et complète de prévention des sinistres informatiques.

\subsection{Pertes financières directes}

Bien que les pertes financières directes ne soient pas l'objectif de cette recherche, il est possible de mettre en évidence les points suivants :

- l'utilisation de l'informatique amène des risques de pertes directes particulièrement dans les applications de contrôle de processus industriels, de gestion financière et commerciale et de gestion du personnel et de la production;

- un système informatique centralisé amène un plus haut niveau de risques qu'un système décentralisé (réparti) ;

- une partie non négligeable (plus de $33 \%$ ) des risques de pertes financières directes est assumée par l'entreprise elle-même (pour une large part, selon nous, parce que ces risques sont mal mesurés par les assurés) ; une faible part de ces risques (11\%) semblent convenablement assurés; cependant nous pensons que la moitié au moins des pertes directes potentielles ne sont couvertes qu'à environ $60 \%$ de leur valeur réelle.

\subsection{Pertes indirectes}

Les pertes indirectes ont été étudiées sous les rubriques suivantes :

- pertes d'exploitation indirectes ;

- responsabilité civile d'exploitation;

- responsabilité civile professionnelle ou contractuelle;

- responsabilité produits ;

- responsabilité civile du détenteur d'information.

Il apparâit clairement que, si les systèmes informatiques n'ont pas généré ces différents types de risques (excepté peut-être pour la R.C. professionnelle), ils ont, par contre, nettement augmenté le niveau de risque existant dans les organisations de type manuel, et apporté des risques nouveaux (défaillance des équipements automatiques, par exemple).

Sur la base de l'échantillon étudié, on peut constater que :

- une entreprise sur six environ n'a même pas couvert les risques de base tels que pertes d'exploitation indirectes, R.C. d'exploitation ;

- au moins une entreprise sur deux n'a pas couvert les risques inhérents à la R.C. professionnelle, à la R.C. produits et à la R.C. détention d'information. 
L'estimation des taux de couverture global, défini comme :

limites supérieures des montants assurés

montant global des sinistres encourus

montre, surtout pour les applications de gestion, une sous-estimation considérable des montants assurés, en particulier due au fait que les systèmes informatiques accroissent les risques anciens et apportent des risques nouveaux.

On peut globalement considérer, en tenant compte d'une relative fiabilité des résultats obtenus avec l'échantillon d'étude, que l'ampleur des sinistres potentiels peut être évaluée à au moins trois fois le montant des assurances prises.

Tout en considérant que le phénomène d'auto-assurance réduit sensiblement le potentiel évoqué ci-dessus, il nous apparaît relativement réaliste d'évaluer le marché potentiel à assurer à environ le double de celui qui existe à l'heure actuelle.

Il peut être noté que :

- les entreprises du secteur industriel sont mieux couvertes que celles des autres secteurs étudiés ;

- de façon générale, les entreprises du Royaume-Uni, de la République fédérale d'Allemagne, de la Suisse et de la Scandinavie ont des taux de couverture nettement supérieurs à ceux des entreprises des autres pays étudiés ;

- l'amplitude du niveau de risque est environ le double pour les applications de type contrôle de processus (maximum : 25 millions de francs suisses; moyenne : 5 millions de francs suisses) que pour les applications de gestion (respectivement 15 et 2 millions de francs suisses);

- $80 \%$ des entreprises de l'échantillon ont déjà connu un ou plusieurs sinistres du type étudié ;

- l'étude des pertes indirectes par type d'incidents indique les valeurs suivantes :

- équipements

$47,4 \%$

- méthodes d'utilisation

$31,4 \%$

- utilisation

$7,5 \%$

- divers (fraudes, sabotages)

$13,7 \%$

- le secteur industriel est potentiellement porteur du plus haut niveau de risques

(en millions de francs suisses)

Max. Médian

industrie

25

services

25

3,5

commerce

3

1,7

0,3

- le niveau de risque est d'autant plus important que les systèmes informatiques sont évolués : les plus fortes pertes indirectes potentielles sont enregistrées dans les systèmes en temps réel ;

- les pertes indirectes potentielles affectent les dommages :

- aux personnes physiques $26,8 \%$

- à l'entreprise elle-même $41 \%$ 
- aux personnes morales clientes $28,1 \%$

- divers $4,1 \%$

soit presque $60 \%$ dans le domaine de la responsabilité civile.

\subsection{Problèmes divers rencontrés pendant l'étude}

- Dans le secteur industriel, un accent tout particulier a été porté sur l'accroissement des risques «Produits»; les participants demandent que les assureurs les soutiennent dans la mise sur pied d'une politique raisonnable et économiquement saine.

- Il est demandé un effort de rationalisation des différentes législations existantes.

La dépendance croissante de notre société vis-à-vis des systèmes informatiques requiert une meilleure appréciation des dangers réels encourus et la mise en œuvre d'une série de mesures concrètes à la fois pour prévenir et pour assurer les risques.

L'industrie de l'assurance peut et doit être l'élément actif des phases de réflexion et d'actions nécessaires.

\section{Méthodes de travail}

Les principales phases de travail, effectuées entre mars 1978 et mars 1979, ont suivi une séquence qui a permis de :

- réfléchir, de manière générale, sur le problème des pertes, directes et indirectes, induites par l'informatique ;

- définir un canevas d'enquête ;

- effectuer les enquêtes et obtenir les informations de base ;

- effectuer une première synthèse ; discuter et valider les résultats obtenus ;

- réaliser un complément d'enquête et effectuer les études de cas prévues ;

- réaliser le rapport final de conclusions.

\subsection{Positionnement du problème}

Deux groupes de 12 et 11 personnes respectivement ont été réunis :

- le premier comprenant des responsables d'entreprises et des directeurs informatiques ;

- le second formé par des spécialistes de l'assurance informatique et des responsables des problèmes d'assurances dans les entreprises.

Ces réunions ont permis en particulier de :

- faire le point sur les divers problèmes rencontrés par assurés et assureurs dans le domaine des pertes potentielles induites par l'utilisation de l'informatique ;

- définir un vocabulaire, de manière à établir une Notice Explicative jointe aux questionnaires d'enquêtes qui devait permettre d'attirer l'attention des répondants sur l'importance des problèmes posés et d'aider ces répondants à fournir les informations utiles à la poursuite de l'étude.

En particulier, pour clarifier les réponses, les rubriques :

- pertes financières :

- frais de reconstitution, 
- frais de dépannage,

- pertes de matières,

- pertes d'exploitation directes,

- pertes d'exploitation indirectes;

- responsabilité civile :

- RC d'exploitation,

- RC professionnelle ou contractuelle,

- RC produits,

- RC du détenteur de l'information, étaient recensées et explicitées.

Dans cette même Notice Explicative, les applications et les incidents possibles étaient répertoriés et codifiés de manière à faciliter, à la fois le travail des répondants et les tâches de dépouillement et de synthèse.

\subsection{Enquêtes}

Le questionnaire mis au point a été envoyé à plus de 300 entreprises européennes.

Par suite des difficultés rencontrées dans l'obtention des informations demandées (en particulier dans l'évaluation des pertes potentielles réelles, et non pas dans la transcription pure et simple des montants assurés), il a été nécessaire de fournir un effort important d'assistance aux entreprises intéressées, voire parfois de réaliser directement l'enquête.

La centaine de réponses valides reçues permet de conclure à une bonne fiabilité des résultats obtenus.

La structure de l'échantillon des répondants est la suivante :

- par situation géographique (en \% du nombre de réponses) :

- France 21

- RFA 14

- Royaume-Uni 18

- Italie 12

- Bénélux 9

- Espagne 9

- Suisse 9

- Scandinavie 8

- par secteur économique (en \% du nombre de réponses) :

- industrie process 16

assemblage $\quad 33$

- commerce 11

- banques - assurances 20

- services 20

- par taille d'entreprises :

Il faut remarquer que, de façon générale, les entreprises contactées se situaient au niveau des grandes entreprises et, pour la plupart, parmi les 50 plus grandes affaires de chacun des pays concernés. 
L'échantillon des répondants se situe parmi le niveau supérieur des entreprises contactées ; les résultats de l'enquête correspondent donc plutôt à la situation des affaires de grande taille au plan européen :

- $55 \%$ des entreprises répondantes ont plus de 10000 employés,

- $25 \%$ ont entre 5000 et 10000 employés,

- $15 \%$ ont entre 1000 et 5000 employés,

- $5 \%$ ont moins de 1000 employés mais se situent dans des créneaux particuliers où elles se placent généralement dans les toutes premières affaires de leur secteur dans leur pays ;

- par type d'utilisation de l'informatique :

- $100 \%$ d'entre elles sont informatisées en gestion,

- $23 \%$ utilisent l'informatique en contrôle de processus.

Encore ici, l'échantillon des répondants peut donc être considéré comme très caractéristique de grandes affaires ayant une grande expérience informatique. Notamment sous l'angle du contrôle de processus puisque plus d'un tiers des firmes répondantes du secteur de l'industrie de l'assemblage ont déjà des réalisations informatiques dans ce domaine alors que le ratio pour les 500 premières entreprises européennes de ce secteur est de $22 \%$ au 31.12.78.

- par ordinateurs employés :

- grands systèmes (prix achat supérieur à 3,5 millions de F.S.) seuls $15 \%$

- grands + moyens et minis systèmes $75 \%$

- moyens et minis systèmes seuls $10 \%$

— par type de systèmes de traitement :

- par lot uniquement $13 \%$

- par lot et télétraitement $72 \%$

- télétraitement uniquement $15 \%$

A noter que $22 \%$ des répondants ont un réseau téléinformatique particulier.

- par catégories de répondants au questionnaire :

- informaticiens $4 \%$

- responsables assurances $2 \%$

- responsables assurances + informaticiens $71 \%$

- responsables assurances + informaticiens + autres répondants $23 \%$

- enfin, il est à noter que $31 \%$ des firmes répondantes, hors sociétés de services spécialisées en informatique, fournissent de l'énergie ou des prestations informatiques à l'extérieur de la société, pour une large part d'ailleurs à l'intérieur de leur groupe.

\subsection{Etude de cas}

Trois études de cas ont été conduites dans trois pays européens différents.

\subsection{Validation qualitative}

Après une synthèse rapide des résultats obtenus dans les phases de recherche précédentes, deux réunions de groupe ont permis de discuter des problèmes de risques induits par l'utilisation des systèmes informatiques. 
Il est alors nettement apparu, quels que soient les types de participants (directeurs d'entreprises, informaticiens, responsables d'assurances dans les entreprises...), les nationalités, les secteurs économiques et la taille des affaires représentées, que :

- si l'assurance des pertes d'exploitation directes est maintenant bien appréhendée, la prévention des incidents possibles n'est pas encore suffisamment complète ;

- et que la responsabilité civile du fait de l'utilisation de l'informatique est très mal connue, est mal mesurée en termes de risk management et que, en particulier, la plupart des participants n'ont jamais été directement confrontés avec une méthode rationnelle d'évaluation des différents niveaux de risques et de choix des solutions possibles.

Quoi qu'il en soit, les idées émises durant toutes les réunions ont été utilisées dans la suite du présent rapport pour essayer de mettre en évidence l'ampleur de la tâche qui reste à accomplir, ainsi que les différents problèmes particuliers à résoudre.

\subsection{Synthèse finale}

Avec les informations détaillées obtenues à partir des questionnaires et des diverses réunions de groupe, il a été possible de développer les conclusions du présent rapport.

Nous devons cependant attirer l'attention des lecteurs de ce rapport sur le point suivant: l'échantillon de réponses reçues n'est pas statistiquement représentatif de l'univers global des entreprises européennes, mais reflète certainement la réalité des problèmes posés par l'utilisation de l'informatique dans les grandes affaires. Il semble cependant qu'on puisse extrapoler les résultats, faits et chiffres obtenus à l'ensemble des secteurs étudiés en considérant toutefois que, plus la taille de l'entreprise est faible, plus il est à craindre que la prise de conscience des risques encourus, et par conséquent la couverture des risques, diminuent, donc qu'augmente la vulnérabilité générale de larges secteurs de l'économie des pays considérés.

\section{Pertes directes}

Bien que l'étude ait été focalisée sur les pertes indirectes, il nous a semblé intéressant et opportun de demander aux répondants de fournir une série d'informations concernant l'évaluation des pertes directes.

Cet élargissement du sujet répondait en fait à deux buts précis :

- d'une part, il permettait de couvrir l'ensemble des problèmes de risques induits par les systèmes informatiques, et d'arriver graduellement, pour les répondants, à la réflexion sur les pertes indirectes par le biais des pertes d'exploitation indirectes avant d'aborder les problèmes de responsabilité civile ;

- d'autre part, nous pouvions ainsi recueillir les informations de base susceptibles d'être utilisées dans une synthèse rapide sur le problème de la sécurité générale des systèmes informatiques et sur celui de l'assurance des pertes directes en particulier.

\subsection{Sécurité générale des systèmes informatiques}

- Dans ce domaine, nous n'avons pas trouvé de différence significative entre les répondants des divers pays étudiés, bien qu'on puisse percevoir un niveau moyen 
de sécurité un peu plus complet en Scandinavie et en R.F.A. ; cependant la taille de l'échantillon ne permet pas une conclusion suffisamment formelle.

- L'analyse par secteur d'activité montre, par contre, une différence notable entre les différents secteurs économiques considérés :

- le secteur banques-assurances apparaît comme ayant réalisé les meilleurs systèmes de sécurité : protection/prévention et possibilités de reprise des activités. Cependant, certaines différences sensibles apparaissent dans ce secteur, aussi bien dans le domaine bancaire que dans celui de l'assurance ;

- le secteur des services peut être également considéré comme relativement bien protégé, notamment parce que les grandes sociétés de service considèrent les systèmes informatiques comme l'outil de production;

- de façon générale, la sécurité informatique paraît faible dans le secteur de l'industrie en particulier pour l'industrie de l'assemblage. Il semble que beaucoup d'entreprises industrielles aient pris le parti de ne considérer que des sinistres peu importants et de n'avoir donc mis en place, en général, que des mesures et méthodes minimales, en particulier sur le plan du système global (équipements et logiciel) qui, très souvent «taillé sur mesure » pour l'entreprise, est réputé quasiment indépannable (ce qui d'ailleurs n'est pas l'avis des spécialistes) ;

- dans le secteur commerce, il nous est apparu que les systèmes de sécurité pouvaient être souvent considérés comme insuffisants.

Degré de sécurité générale atteint par secteur d'activité

\begin{tabular}{|ll|c|c|c|c|}
\hline & & 1 & 2 & 3 & 4 \\
\hline Industrie & $\begin{array}{l}\text { Process } \\
\text { Assemblage }\end{array}$ & $60 \%$ & $65 \%$ & - & $50 \%$ \\
\cline { 2 - 6 } & $50 \%$ & $60 \%$ & - & $55 \%$ \\
\hline Commerce & $40 \%$ & $50 \%$ & - & $60 \%$ \\
\hline Banque-Assurances & $70 \%$ & $92 \%$ & $5 \%$ & $40 \%$ \\
\hline Services Divers & $75 \%$ & $98 \%$ & $27 \%$ & $30 \%$ \\
\hline
\end{tabular}

1) Existence d'une procédure formalisée de sauvegarde.

2) Existence d'un contrat formalisé de dépannage.

3) Existence d'une validation périodique des procédures de sécurité.

4) Existence d'un niveau de sinistre ne permettant pas la reprise immédiate des activités.

- L'existence d'une procédure précise et formalisée de sauvegarde en cas de sinistre important n'existe pas dans environ un tiers des cas étudiés (se référer au tableau précédent pour un détail par secteur d'activité).

- Le contrat de dépannage (back-up) permettant à l'utilisateur d'avoir recours à un autre équippement pour traiter ses propres applications en cas de sinistre grave est fréquent (environ $75 \%$ des répondants). 
Cependant, cette possibilité présente des limites importantes :

- Le dépannage de systèmes en temps réel où les interfaces centre de traitement - réseau - terminaux sont très spécifiques à chaque entreprise pose des problèmes de transfert quasi insurmontables.

- La vulnérabilité des logiciels/fichiers demeure très haute; prévoir une possibilité de dépannage au niveau des équipements sans une protection complète des logiciels et des fichiers s'avère une illusion.

- La validation effective du système de sécurité par essai périodique n'est pratiquement jamais mise en œuvre, sauf dans les sociétés de service informatique.

Or, il est bien évident que les procédures de travail évoluent, les matériels et les logiciels également, et que seule une procédure systématique de vérification périodique peut garantir que le système de sécurité est toujours bien adapté aux besoins de l'entreprise.

Il semble cependant qu'il serait aussi de l'intérêt des assureurs de conseiller, voire d'exiger, au moins un exercice de ce type chaque année pour vérifier que les bases de calcul des risques, et des primes, sont toujours valables.

- Plus de la moitié des répondants pensent qu'il existe un niveau de sinistre qui ne permet pas une reprise des activités de l'entreprise.

En fait, un contrôle détaillé permet de correler quasi parfaitement la réponse positive à cette question et le degré de sécurité général du système informatique.

Les entreprises qui admettent un niveau de sinistre irrémédiable sont, en général, celles qui présentent un faible taux de sécurité globale et qui semblent, par là même, justifier une fatalité irréversible.

Synthèse :

- Il doit être possible d'améliorer largement la sécurité des systèmes informatiques, notamment dans le domaine de l'industrie et du commerce.

- Il semble que la responsabilité de cet état de fait incombe aussi bien aux assurés qu'aux assureurs ; les uns n'attachent sans doute pas toute l'importance nécessaire à ce problème ; quant aux seconds, ils n'incitent sans doute pas (ou pas suffisamment) leurs clients à prendre les dispositions nécessaires.

- Un effort méthodologique formalisé (dossiers, procédures, audit) paraît nécessaire pour que la sécurité des systèmes informatiques soit convenablement traitée.

\subsection{Evaluation des pertes financières directes}

\subsubsection{Définition des risques}

Dans ce domaine, comme dans d'autres types de risques, les entreprises sont confrontées avec les problèmes de définition d'un niveau de risque :

- en dessous duquel l'entreprise prend à sa charge les pertes financières qui découlent des incidents ;

- au-dessus duquel l'entreprise a recours à l'industrie de l'assurance pour faire face aux pertes financières. 
De façon quasi générale, en informatique, la définition de ce niveau de risque n'est pas claire :

- pour des motifs d'efficacité (les procédures de déclaration d'incidents sont réputées lourdes, les processus d'accord entre assurances/assurés sont longs et compliqués... disent les assurés) ainsi que, sans doute pour éviter une mise en lumière d'incidents peu importants mais nombreux, les informaticiens prennent à leur compte les risques de fréquence forte mais d'amplitude faible (quelques centaines de francs suisses) et s'assurent pour les risques importants (incendie, dégâts des eaux) même si cette attitude n'est pas toujours valable sur le plan économique.

\subsubsection{Risques}

Frais de reconstitution

Frais de dépannage

Pertes de Matières

Pertes d'exploitation directes

\section{Assurés}

$80 \%$ des cas

$90 \%$ des cas

$50 \%$ des cas

$30 \%$ des cas
Existants réellement

$100 \%$

$100 \%$

$66 \%$

$100 \%$

\subsubsection{Applications}

Les principales applications justifiant une assurance des pertes financières directes, par ordre décroissant d'importance :

- automatisation de processus

- gestion financière

- gestion commerciale

- gestion du personnel

- gestion de production

$100 \%$ des cas

$85 \%$ des cas

$85 \%$ des cas

$72 \%$ des cas

$72 \%$ des cas

- gestion distribution

- gestion de banques de données

$58 \%$ des cas

$44 \%$ des cas

Enfin, dans les secteurs finances et commerce, les applications :

- gestion de portefeuilles,

- vente par correspondance

présentent également un haut niveau de risques potentiels.

\subsubsection{Incidents}

Pour évaluer l'importance des incidents potentiels, il nous est apparu utile de rechercher une unité de mesure qui puisse faire apparaître le niveau de risque induit par type d'incident. Dans cet esprit, nous avons considéré que l'unité de mesure la plus satisfaisante était celle qui combinait à la fois le niveau de pertes directes par incident et la fréquence annuelle estimée de l'incident.

Le ratio risque relatif, dont l'analyse chiffrée figure ci-dessous, est donc défini comme le produit de la part du montant total de l'assurance pertes directes souscrite par l'entreprise (ce montant total étant affecté de la base 100) par la fréquence annuelle d'incidents.

\section{Exemple :}

- Une entreprise a souscrit une assurance pertes directes pour un montant de 1000000 de F.S. (base 100). 
- Une erreur d'exploitation (fréquence annuelle 2) induit un sinistre d'un montant de 100000 F.S. (soit 1/10 de la base 100, c'est-à-dire 10).

- Risque relatif : $10 \times 2=20$.

Ceci permet donc d'établir, après analyse, une liste correspondant, en moyenne, au montant relatif des pertes par types d'incidents pour une entreprise de l'échantillon :

- arrêt momentané du système

$19 \%$

- pertes ou destruction de fichiers

$15 \%$

- erreurs d'exploitation

$14 \%$

- erreurs de codage en entrée

$13 \%$

- arrêt total du système

$11 \%$

- erreurs de programmation

$9 \%$

- parures de terminaux

$8 \%$

- fraudes - sabotages

$7 \%$

- divers

$4 \%$

Ceci signifie que, pour une entreprise utilisant l'informatique, il serait nécessaire de couvrir tous les risques inhérents aux incidents ci-dessus.

Or, en réalité, une analyse des contrats existants montre, qu'à l'heure actuelle et sur l'échantillon considéré, la couverture des risques inhérents aux incidents se présente comme suit :

- arrêt momentané du système

- pertes ou destruction de fichiers

- erreurs d'exploitation

- erreurs de codage en entrée

- arrêt total du système

- erreurs de programmation

- pannes de terminaux

- fraudes - sabotages

- divers

\begin{tabular}{|c|c|c|}
\hline Totale & Moyenne & Nulle \\
\hline$\times$ & $\times$ & \\
& & $\times$ \\
$\times$ & & \\
& & $\times$ \\
$\times$ & $\times$ & \\
& $\times$ & \\
\hline
\end{tabular}

On voit donc rapidement que :

- $36 \%$ des risques relatifs ne sont pas du tout couverts;

$-27 \%$ le sont à environ $50 \%$;

- $26 \%$ le sont à $75 \%$;

- $11 \%$ paraissent l'être convenablement.

5.2.5 Niveau de risque et type de système informatique utilisé

Il paraît évident aux informaticiens qu'un système centralisé travaillant en temps réel avec un réseau de télécommunications est beaucoup plus vulnérable et présente un beaucoup plus haut niveau de risques qu'un système décentralisé travaillant par lots. 


\subsubsection{Synthèse}

La couverture réelle totale des pertes financières induites par l'utilisation des systèmes informatiques est faible : un bon tiers des risques est assuré par l'entreprise elle-même ; la moitié est assurée pour environ $60 \%$ de la valeur réelle. Seuls $19 \%$ semblent convenablement assurés.

\section{Pertes indirectes}

L'étude a pour objectif essentiel de déterminer la nature et l'importance des risques à couvrir du fait de l'utilisation des systèmes informatiques au niveau :

- des pertes d'exploitation,

- de la responsabilité civile,

et de donner, en conclusion, les différentes recommandations et informations utiles permettant aux responsables de réfléchir à leur stratégie dans ce domaine.

\subsection{Définitions adoptées}

Nous avons séparé les pertes indirectes en :

- pertes d'exploitation indirectes :

- pertes de contrats, pertes de clientèle permanente,

- frais financiers induits, du fait de l'utilisation de l'informatique ;

— responsabilité civile d'exploitation :

- couvrant les dommages causés à des tiers du fait d'une mauvaise utilisation de l'informatique dans l'exploitation de l'entreprise ;

- responsabilité civile professionnelle ou contractuelle :

- qui s'applique aux entreprises fournissant des services informatiques (sociétés de services ou prestataires dont ce n'est pas l'activité principale) pour les dommages causés à des tiers par le fonctionnement défectueux de l'informatique ;

- responsabilité produits :

- étudiée sous l'angle des dommages induits par des produits non conformes, fabriqués et/ou livrés aux clients après utilisation directe de l'informatique dans l'une quelconque des étapes du processus de conception - développement - fabrication - distribution ;

- responsabilité civile du détenteur de l'information :

- qui couvre les dommages induits par la divulgation d'informations confidentielles traitées par les systèmes informatiques.

Il est bien évident que l'informatique n'est pas à la base de ces différents types de risques (excepté pour la R.C. professionnelle ou contractuelle) par exemple :

- un système de comptabilité manuelle peut générer des erreurs préjudiciables à un client : une banque européenne a été condamnée en 1964 pour avoir bloqué pendant plusieurs mois, par erreur, le compte d'un de ses clients (dans ce cas, les traitements étaient effectués par une organisation utilisant des matériels de type comptable) ;

- les erreurs de dosage d'une matière dangereuse dans un produit français étaient occasionnés par un réglage manuel ; l'assureur a réglé plus de 10 millions de francs de dommages et intérêts ; 
- la divulgation d'informations confidentielles par une banque européenne a été lourdement condamnée en 1968 ; pourtant les employés indélicats s'étaient servis de listes éditées au sein du département "gestion des fortunes».

Cependant, nous avons recherché dans la présente étude si l'utilisation extensive de l'informatique n'avait pas créé de nouvelles conditions de fonctionnement dans l'exploitation des entreprises et si ces nouvelles conditions de fonctionnnement n'avaient pas engendré de nouveaux risques et/ou augmenté les risques existants.

\subsection{Méthode de travail}

C'est pourquoi nous avons demandé aux répondants de travailler :

- application par application ;

- et pour chacune d'entre elles, par type d'incidents;

de manière à pouvoir, en synthèse, déterminer quel était le niveau réel estimé des pertes indirectes, rubrique par rubrique, et évaluer enfin si la couverture actuelle des entreprises était suffisante ou non.

\subsection{Rappel sur la sécurité générale des systèmes informatiques}

Il est évident que le problème des pertes indirectes est lié de façon étroite à la sécurité générale des systèmes informatiques. Nous sommes donc, globalement, placés devant deux hypothèses possibles.

\section{Hypothèse no 1}

$\mathrm{Si}$ les systèmes informatiques étaient réputés fiables à $100 \%$, le niveau des pertes indirectes induites par l'utilisation de l'informatique tendrait vers zéro ; étant donné que l'informatique pénètre de plus en plus profondément dans le fonctionnement des entreprises, le risque global de pertes indirectes de l'entreprise diminuerait sensiblement.

\section{Hypothèse $n^{\circ} 2$}

$\mathrm{Si}$, au contraire, la sécurité générale des systèmes informatiques est mauvaise et/ou moins élevée que la sécurité d'un système manuel, l'utilisation extensive de l'informatique pour l'entreprise doit conduire à une augmentation du risque global de pertes indirectes par cette entreprise.

Il nous faut donc rappeler ici brièvement les principales conclusions de l'enquête (cf. chapitre 5.1) sur la sécurité générale des systèmes informatiques :

- niveau de sécurité générale peu satisfaisant, en particulier dans les secteurs économiques de l'industrie et du commerce ;

- effort de rationalisation nécessaire pour assurer un niveau de sécurité compatible avec l'importance fonctionnelle des systèmes informatiques.

En conséquence, il apparaît que, d'ores et déjà, nous pouvons situer qualitativement le problème, de manière générale, dans le cas de l'hypothèse $n^{\circ} 2$ :

- les entreprises européennes, ayant de plus en plus recours à l'informatique, et la sécurité générale des systèmes informatiques étant médiocre, le risque global des pertes indirectes a tendance à croître en corrélation avec l'informatisation de l'entreprise. 
6.4 Estimation globale des taux de couverture des pertes indirectes

Principe d'évaluation:

Les réponses obtenues aux questions concernant les différentes applications identifiées par les répondants à l'enquête (voir chapitre suivant 6.5 pour l'analyse de détail) ont permis de déterminer, pour chaque entreprise :

- le type de pertes indirectes potentielles et l'existence ou non de la couverture du risque correspondant;

- une estimation du taux de couverture global de ce risque pour l'entreprise, ventilée par nature d'utilisation de l'informatique :

- applications de gestion,

- applications de contrôle de processus.

La synthèse ci-dessous présente donc, par type d'assurance de pertes indirectes :

- le pourcentage d'entreprises qui ont couvert chacun des risques étudiés par un contrat d'assurance ;

- pour les entreprises ayant participé à l'étude, une estimation de la couverture globale, en valeur, des assurances souscrites par rapport au montant global des sinistres encourus.

Taux de couverture global :

Limites supérieures des montants assurés

Montant global des sinistres encourus

Estimation des taux de couverture actuels des pertes indirectes

\begin{tabular}{|l|c|c|c|}
\hline \multicolumn{1}{|c|}{ Type d'assurance } & $\begin{array}{c}\text { Pourcentage assuré de } \\
\text { l'échantillon }\end{array}$ & \multicolumn{2}{|c|}{$\begin{array}{c}\text { Taux de couverture } \\
\text { global }\end{array}$} \\
\hline & & Gestion & $\begin{array}{c}\text { Contrôle } \\
\text { de } \\
\text { processus }\end{array}$ \\
\hline Pertes d'exploitation & $88 \%$ & $50 \%$ & $60 \% *$ \\
RC professionnelle ** & $40 \%$ & $10 \%$ & $20 \%$ \\
RC d'exploitation & $80 \%$ & $15 \%$ & $40 \%$ \\
RC produits *** & $45 \%$ & $10 \%$ & $35 \%$ \\
RC détention d'information ** & $40 \%$ & $50 \%$ & - \\
\hline
\end{tabular}

* Avec une grande amplitude dans les estimations obtenues (de 20 à $85 \%$ pour les bornes mini-maxi).

** Valeurs estimées pour les entreprises susceptibles de contracter :

- une RC professionnelle : services bureaux et entreprises cédant de l'énergie informatique ;

- une RC détention d'information : toutes entreprises utilisant des fichiers susceptibles de conserver des informations confidentielles.

*** Valeurs estimées pour les entreprises susceptibles de contracter une RC produits. 
En conclusion, les résultats montrent :

- une proportion non négligeable d'entreprises (de 12 à $20 \%$ ) n'ayant même pas couvert les risques principaux concernant :

- les pertes d'exploitation indirectes,

- la RC d'exploitation.

Certaines des entreprises ayant répondu à l'enquête ont souligné qu'il était vraisemblable que l'entreprise ait décidé d'assurer elle-même ces pertes indirectes; aucune d'entre elles n'a trouvé une référence précise à cette décision ;

- plus de $50 \%$ des entreprises concernées (voir remarques sous le tableau précédent) ne sont pas couvertes pour les risques :

- RC professionnelle,

- RC produits,

- RC détention d'information;

- pour les applications de gestion, le taux de couverture global est très faible, de 10 à $50 \%$, en particulier pour la RC produits. La quasi-totalité des répondants a en effet considéré que l'utilisation de l'informatique apportait des risques nouveaux et qu'il y avait donc, sur ce point, une sous-estimation actuelle considérable, en particulier parce que le problème n'a jamais été considéré, à la fois globalement (risk management) et à la fois de façon détaillée ;

- au niveau des applications de contrôle de processus le taux de couverture est meilleur, quoique encore estimé par les répondants largement insuffisant (35\% pour la $\mathrm{RC}$ produits et $60 \%$ pour les pertes d'exploitation indirectes);

- l'analyse par secteur économique montre, de façon générale, que les entreprises industrielles et les entreprises pour qui l'informatique constitue l'activité principale sont un peu mieux couvertes que les entreprises des autres secteurs économiques étudiés, et en tout cas, paraissent plus ouvertes à ces concepts que les entreprises du secteur finance et surtout que celles du secteur distribution, où il apparaît que le marché à prendre peut être considéré comme le triple ou le quadruple du marché existant ;

- l'analyse par pays semble indiquer une distorsion importante entre les entreprises :

- d'une zone « Nord-Europe » (Royaume-Uni, RFA, Scandinavie),

- et de la zone « Pays Latins » (France, Espagne, Italie), où les valeurs résultantes obtenues sont très sensiblement inférieures.

\subsection{Analyse par applications}

Les réunions de groupe effectuées au départ de l'enquête ont permis d'identifier une liste d'applications de l'informatique (divisées en deux parties: applications de gestion et applications de contrôle de processus) paraissant susceptibles d'induire un haut niveau de risques de pertes indirectes.

La liste de ces applications a été fournie aux répondants à l'enquête, soit :

- 16 applications de gestion;

- 11 applications de contrôle de processus.

Pour chacune d'entre elles correspondant à l'utilisation réelle de l'informatique dans l'entreprise, les répondants devaient préciser pour les trois types d'incidents les 
plus significatifs, les montants des pertes financières (directes et indirectes) et des dommages de responsabilité civile suivant les schémas ci-dessous :

\begin{tabular}{|c|c|}
\hline & Application \\
\hline \multirow{2}{*}{$\begin{array}{c}\mathrm{I} \\
\mathrm{N}\end{array}$} & Frais de reconstitution \\
\hline & Frais de dépannage \\
\hline 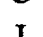 & Pertes de matières \\
\hline D & $\begin{array}{l}\text { Pertes d'exploitation } \\
\text { directes }\end{array}$ \\
\hline $\begin{array}{l}\mathbf{N} \\
\mathrm{T}\end{array}$ & $\begin{array}{l}\text { Pertes d'exploitation } \\
\text { indirectes }\end{array}$ \\
\hline
\end{tabular}

\begin{tabular}{|l|l|}
\cline { 2 - 2 } \multicolumn{1}{c|}{} & \multicolumn{1}{c|}{ Application } \\
\hline I & Dommages corporels \\
N & C \\
I & Dommages matériels \\
D & Dommages immatériels \\
E & N \\
T & Type de responsabilité \\
\hline
\end{tabular}

\subsubsection{Amplitude des pertes indirectes}

Les figures 1 et 2 montrent les estimations faites par les répondants de l'amplitude moyenne et de l'amplitude maximale pour chacune des applications retenues pour l'analyse.

Ces résultats appellent plusieurs commentaires importants :

- Le niveau de risque est nettement plus important pour les applications de type contrôle de processus que pour celles de type gestion : en moyenne le rapport des niveaux de risque entre les deux catégories d'application est de 2 à 1 .

- On peut considérer que le niveau de risque total pour une entreprise est équivalent à la somme des risques afférents à chacune des applications particulières traitées par l'entreprise en informatique. Cependant, la situation spécifique de chaque entreprise requiert un examen précis de chaque application, de son niveau de risque (par rapport à la taille de l'entreprise, à son secteur économique) avant d'en tirer une évaluation globale.

L'intérêt principal des informations obtenues ici doit se situer dans l'utilisation conceptuelle et pratique qui peut en être faite, par exemple :

- au niveau stratégique et politique du secteur de l'assurance : faire réexaminer les contrats de R.C. dans les firmes qui utilisent l'informatique pour la conception de produits nouveaux, ou les firmes pharmaceutiques qui gèrent leur production par informatique; mettre en place une politique et une stratégie spécifique pour promouvoir l'assurance R.C. dans tel ou tel secteur faisant un important recours à l'informatique pour telle application (exemple : gestion de portefeuille dans les organismes financiers);

- au niveau pratique, attirer l'attention de tous ceux (agents, responsables divers) qui sont confrontés à l'évaluation du niveau de risque de pertes indirectes dans une entreprise.

- Quoique l'étude n'ait porté que sur les trois incidents les plus importants pour chacune des applications, on peut considérer qu'on a obtenu ici l'amplitude maximale correspondant bien à la perte maximale subie par l'entreprise, y compris dans 
le cas de survenance de plusieurs incidents quasi simultanés : l'un de ces incidents étant toujours dominant par rapport aux autres (par exemple, dans le secteur distribution, la destruction complète du fichier amène une perte plus importante que l'incendie total de l'ordinateur lui-même).

Fig. 1 : Amplitude maximale et moyenne par application et par entreprise (en milliers de francs suisses)

\begin{tabular}{|c|c|c|c|}
\hline & & $\begin{array}{l}\text { Amplitude } \\
\text { maximale }\end{array}$ & $\begin{array}{l}\text { Amplitude } \\
\text { moyenne }\end{array}$ \\
\hline 01 & Gestion du personnel & 250 & 125,5 \\
\hline 02 & Gestion financière & 4500 & 2251,0 \\
\hline 03 & Gestion commerciale & 3000 & 1500,0 \\
\hline 04 & Gestion distribution & 800 & 400,7 \\
\hline 05 & Gestion de flotte & 10000 & 5007,5 \\
\hline 07 & Gestion de portefeuille & 5000 & 2500,8 \\
\hline 09 & Vente par correspondance & 1000 & 500,4 \\
\hline 11 & Gestion de production & 1800 & 903,5 \\
\hline 12 & Contrôle général & 15000 & 7575,0 \\
\hline 20 & Contrôle des raffineries & 5000 & 2525,0 \\
\hline 21 & $\begin{array}{l}\text { Contrôle de fabrication } \\
\text { dans l'industrie alimentaire }\end{array}$ & 5000 & 2537,5 \\
\hline 22 & $\begin{array}{l}\text { Contrôle de fabrication } \\
\text { pharmacie/cosmétique }\end{array}$ & 25000 & 12517,5 \\
\hline 23 & Contrôle de fabrication chimie & 8000 & 4025,0 \\
\hline 24 & Contrôle de fabrication assemblage & 1500 & 787,5 \\
\hline 25 & Commandes de machines-outils & 5000 & 2507,5 \\
\hline 26 & Conception de produits & 25000 & 12550,5 \\
\hline 28 & Contrôle qualité pièces et produits & 一 & - \\
\hline
\end{tabular}


Fig. 2 : Amplitude maximale et moyenne par application et par entreprise (en milliers de francs suisses)

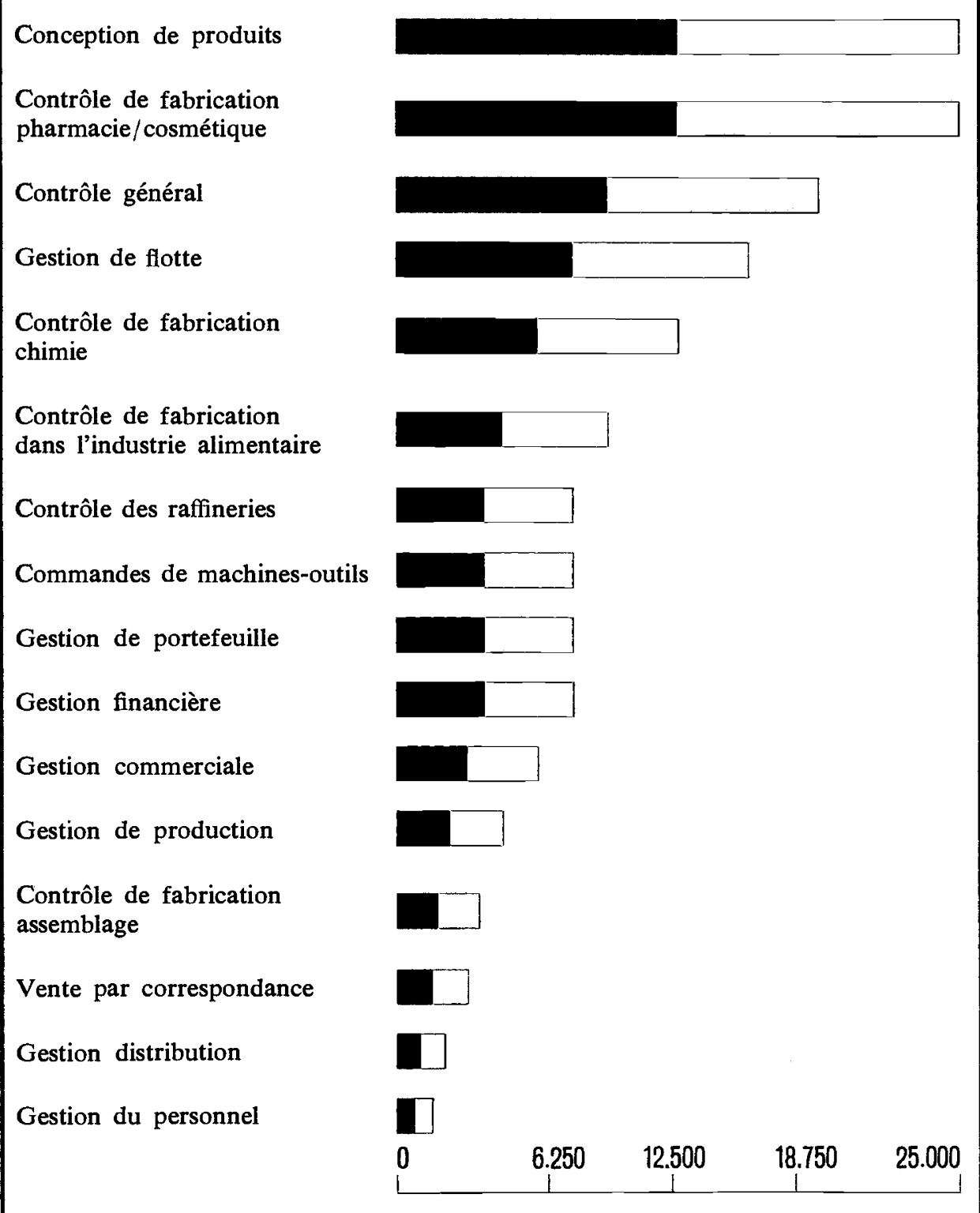


6.5.2 Synthèse des niveaux de pertes indirectes possibles par type d'application

Les réponses obtenues à l'enquête indiquent les niveaux de pertes indirectes possibles, en millions de francs suisses :

- pour les applications de gestion

Max.

Moyen

- pour les applications de contrôle de processus

\subsubsection{Fréquence des pertes indirectes}

Pour rendre la compréhension plus aisée, le tableau ci-après représente la fréquence de pertes indirectes, par entreprise :

- estimée (moyenne obtenue à partir des questionnaires),

- corrigée (après discussion par les groupes de travail),

pour des sinistres définis, suivant leur ampleur, comme :

- lourds (supérieurs à 750000 francs suisses),

- moyens (50 000 à 749999 francs suisses),

- faibles (moins de 50000 francs suisses),

pour les applications de gestion et de contrôle de processus.

Fréquence de pertes indirectes par entreprise

\begin{tabular}{|l|c|c|c|c|}
\hline \multicolumn{2}{|c|}{ Sinistres } & \multicolumn{2}{c|}{ Gestion } & \multicolumn{2}{c|}{ Contrôle de processus } \\
\hline \multirow{2}{*}{ Lourds } & $\frac{\text { Estimée }}{2}$ & $\frac{\text { Corrigée }}{2}$ & $\frac{\text { Estimée }}{2}$ & Corrigée \\
Moyens & 0,04 & 0,03 & 0,02 & 0,02 \\
Faibles & 0,2 & 0,4 & 0,1 & 0,4 \\
& 2,8 & 4 & 0,9 & 1 \\
\hline
\end{tabular}

\subsection{Analyse par type d'incidents}

Une liste d'incidents possibles a été mise au point par les participants aux réunions de groupe et incluse dans le questionnaire diffusé de façon à fournir aux répondants une définition des principaux types d'incidents et un support à leur réflexion.

\subsubsection{Résultats généraux}

La figure 3 montre la répartition relative des pertes indirectes totales (pertes d'exploitation et R.C.) par type d'incidents regroupés en quatre domaines :

- équipement (ordinateurs, pluriphysiques, terminaux);

- méthodes d'utilisation (exploitation et logiciel) ;

- utilisation (erreurs des utilisateurs) ;

- divers (fraudes, sabotages) ;

pour l'échantillon d'étude retenu et pour les deux types d'applications : gestion et contrôle de processus. 
Fig. 3 : Pertes indirectes totales par type d'incidents

\begin{tabular}{|l|c|c|}
\hline & & $\begin{array}{c}\text { \% relatif } \\
\text { - Equipestion + processus } \\
47,4\end{array}$ \\
- Interruption du service & & \\
- Pannes terminaux et périphériques & 23,5 & 23,9 \\
- Méthode d'utilisation & & 31,4 \\
- Logiciel & 11,8 & \\
- Exploitation & 19,6 & 7,5 \\
- Utilisation & & 13,7 \\
- Divers & 11,7 & \\
- Fraudes, sabotages & 2 & 100 \\
- Autres & & \\
\hline
\end{tabular}

Fig. 4: Pertes indirectes totales par type d'incidents

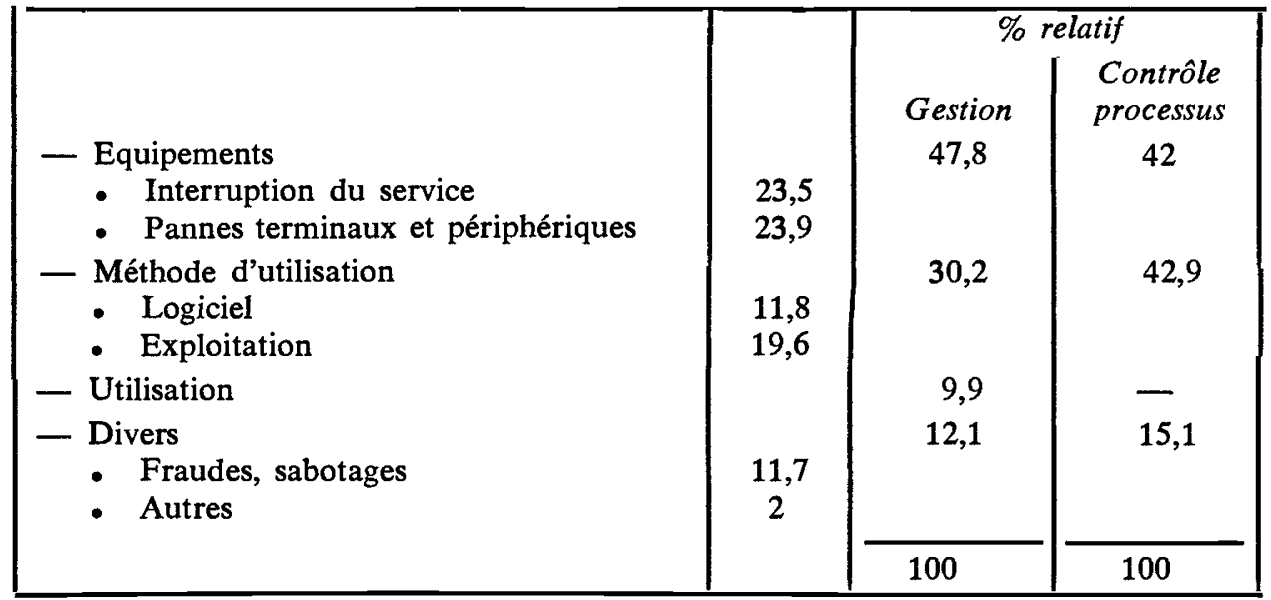

6.7 Analyse des niveaux de pertes indirectes par secteurs économiques étudiés

Le tableau ci-dessous montre, en millions de francs suisses, le montant maximal et médian des pertes indirectes encourues par les entreprises ayant participé à notre enquête

Niveau des pertes indirectes par secteur économique

Industrie Max.

Médian

Services 25

3,5

Commerce

25

1,7

3

0,3

Il est à noter que :

- les estimations concernant le secteur du commerce indiquent, et ceci est particulièrement marqué lorsqu'on analyse le niveau de la R.C. : \& la R.C. produits est 
l'affaire du fabricant $» . .$. , un faible niveau potentiel pour les pertes indirectes, pour la quasi-totalité d'entre elles dans le domaine des pertes d'exploitation indirectes. Cette remarque est valable pour toutes les entreprises de ce secteur ayant répondu à notre enquête ; cependant certains experts considèrent que, en général, les entreprises commerciales ont tendance à sous-estimer les risques encourus (perte de clientèle, par exemple) ;

- dans le domaine des services, l'analyse statistique des réponses obtenues fait apparaître une segmentation géographique assez remarquable :

- les entreprises des pays de «type latin » (ou de droit écrit) estiment, de façon assez générale, que leurs risques en R.C. d'exploitation sont peu importants,

- tandis que, au contraire, les entreprises des pays de «l'Europe du Nord» indiquent des niveaux de risques en R.C. d'exploitation environ deux fois supérieurs, à activité et taille similaire, à ceux des entreprises de l'autre groupe géographique.

Enfin, on peut noter un niveau de risques de pertes indirectes particulièrement élevé pour les entreprises prestataires de services en informatique.

La même remarque est valable dans le secteur industriel encore que les différences constatées soient sensiblement moindres (environ $50 \%$ d'écart) entre les deux groupes géographiques.

\subsection{Analyse des niveaux de pertes indirectes par types de systèmes installés}

Il existe une forte corrélation entre le niveau de pertes indirectes possibles et le type de système informatique installé dans l'entreprise ainsi qu'il est montré dans le tableau ci-dessous :

Temps réel

Lots à distance

Lots
Max.

25

12

4,9
Médian

3

1,4

0,8

\subsection{Analyse par types de dommages}

Après identification sur l'échantillon d'étude :

- du taux de couverture des pertes indirectes;

- des applications potentiellement porteuses de pertes indirectes;

- de l'amplitude et de la fréquence des pertes indirectes;

- des différents types d'incidents possibles;

nous avons évalué, à partir des informations obtenues, quels différents types de dommages étaient pris en compte par les répondants.

Comme dans l'étude précédente réalisée pour l'Association de Genève, les différents types de dommages ont été classés en quatre catégories :

- personnes physiques, qu'elles soient de l'entreprise ou externes à l'entreprise (clients) pour des atteintes à leur santé, leur vie, leur réputation ;

- entreprise elle-même ;

- clients, personnes morales ;

- divers (cas imprécis, Etat et collectivités publiques). 
Le tableau ci-dessous indique la répartition des pertes indirectes potentielles pour l'échantillon d'étude (applications de gestion et de contrôle de processus confondues) :

- personnes physiques

- entreprise elle-même

- clients, personnes morales

- divers

$$
\begin{array}{r}
26,8 \% \\
41 \% \\
28,1 \% \\
4,1 \% \\
\hline 100 \%
\end{array}
$$

Il semble que, dans l'échantillon étudié :

- environ $40 \%$ des pertes indirectes soient le fait de pertes d'exploitation indirectes : pertes de clientèle, de contrats, frais financiers entraînés par le rebut de produits et/ou par la non-satisfaction de la clientèle ;

- plus de $50 \%$ des sinistres soient le fait de la responsabilité civile vis-à-vis de tiers (clients et utilisateurs en particulier).

Bien entendu, l'échantillon d'étude n'est pas suffisant pour en tirer des conclusions définitives mais les résultats ci-dessus, analysés par secteur économique, montrent que, pour les entreprises d'un des secteurs étudiés, le montant de la couverture globale de l'entreprise devait être ventilé entre les deux rubriques considérées, de la façon suivante :

$\begin{array}{lccc} & \text { Industrie } & \text { Finance } & \text { Commerce } \\ \text { Pertes d'exploitation indirectes } & 36 \% & 48 \% & 56 \% \\ \text { Dommages type R.C. } & 64 \% & 52 \% & 44 \%\end{array}$

Il n'a pas été possible d'établir des ratios significatifs au niveau des différents pays concernés par l'étude.

Une ventilation des résultats ci-dessus entre applications de gestion et de contrôle de processus donne les chiffres suivants :

- personnes physiques

- entreprise elle-même

- clients, personnes morales

- divers

\begin{tabular}{cc} 
Gestion & Contrôle de processus \\
18,1 & 29,9 \\
49,2 & 38,7 \\
29,1 & 26,4 \\
3,6 & 5 \\
\hline $100 \%$ & $100 \%$
\end{tabular}

Il reste beaucoup à faire, en particulier dans le domaine des pertes indirectes, pour que la dépendance croissante des entreprises vis-à-vis de l'informatique n'ait pas de conséquences graves en cas de sinistres.

C'est au prix, croyons-nous, d'une collaboration totale entre assurés et assureurs, notamment dans les domaines de :

- la politique de prévention/détection et d'assurance des risques,

- la définition de méthodes à mettre en œuvre, que doit se dégager une pratique réellement efficace de risk-management dans notre société de plus en plus dépendante des systèmes informatiques. 